\title{
BMJ Open Improving outcomes from high-risk surgery: a multimethod evaluation of a patient-centred advanced care planning intervention
}

\author{
Amanda Selwood, ${ }^{1}$ Siva Senthuran, ${ }^{2,3}$ Brette Blakely, ${ }^{1}$ Paul Lane,${ }^{2}$ John North, ${ }^{4}$ \\ Robyn Clay-Williams ${ }^{1}$
}

To cite: Selwood A Senthuran S, Blakely B, et al. Improving outcomes from high-risk surgery: a multimethod evaluation of a patient-centred advanced care planning intervention. BMJ Open 2017;7:e014906. doi:10.1136/bmjopen-2016014906

- Prepublication history and additional material is available. To view please visit the journal (http://dx.doi.org/ 10.1136/bmjopen-2016014906).

Received 25 October 2016 Revised 18 January 2017 Accepted 7 February 2017

CrossMark

For numbered affiliations see end of article.

Correspondence to Dr Amanda Selwood; amanda.selwood@mq.edu.au

\section{ABSTRACT}

Introduction: Patients who are frail, have multiple comorbidities or have a terminal illness often have poor outcomes from surgery. However, sole specialists may recommend surgery in these patients without consultation with other treating clinicians or allowing for patient goals. The Patient-Centred Advanced Care Planning (PC-ACP) model of care provides a framework in which a multidisciplinary advanced care plan is devised to incorporate high-risk patients' values and goals. Decision-making is performed collaboratively by patients, their family, surgeons, anaesthetists,

intensivists and surgical case managers. This study aims to evaluate the feasibility of this new model of care, and to determine potential benefits to patients and clinicians.

Methods and analysis: After being assessed for frailty, patients will complete a patient-clinician information engagement survey pretreatment and at 6 months follow-up. Patients (and/or family members) will be interviewed about their experience of care pretreatment and at 3 and 6 months follow-ups. Clinicians will complete a survey on workplace attitudes and engagement both preimplementation and postimplementation of PC-ACP and be interviewed, following each survey, on the implementation of PCACP. We will use process mapping to map the patient journey through the surgical care pathway to determine areas of improvement and to identify variations in patient experience.

Ethics and dissemination: This study has received ethical approval from Townsville Hospital and Health Service HREC (HREC/16/QTHS/100). Results will be communicated to the participating hospital, presented at conferences and submitted for publication in a peerreviewed MEDLINE-indexed journal.

\section{INTRODUCTION}

Frail, high-risk patients presenting for surgery pose a complex problem. For them, surgery can offer hope, but also the potential for many adverse events. ${ }^{1}{ }^{2}$ Patients who are frail, have a terminal illness or have multiple

\section{Strengths and limitations of this study}

- This study will evaluate the implementation of a new surgical model to improve surgical decisionmaking for high-risk patients in a real-world hospital setting.

- The new surgical model has the potential to improve patient satisfaction and interdisciplinary engagement across specialties, by involving patients, their families and clinicians from various disciplines in decision-making. It also has the potential to decrease the costs of prolonged or inappropriate treatments.

- This evaluation will assess patient and clinician experience of the new surgical model to inform future implementation of the new model.

- Participant attrition will be a key challenge for this study due to the high risk of poor outcomes in the patient population. In order to minimise the effects of attrition, family members may be interviewed on patients' behalf.

- This study is a feasibility evaluation and not a clinical trial. It has a small sample size and will not provide information on the impact of the new surgical model on patient outcomes.

comorbidities have poorer outcomes from surgery than other patients. ${ }^{3-6}$ However, decisions made by a sole specialist do not always take into account the high likelihood of multisystem complications facing these patients. Additionally, during the perioperative period, advance directives tend to be poorly managed, particularly Do Not Resuscitate (DNR) orders for postoperative care. ${ }^{7} 8$ These factors may lead to high-risk patients receiving non-beneficial surgical treatments or treatments that do not align with their goals. ${ }^{9}$

One of the study authors (SS) has developed a decision-making process for surgical patients who are identified prior to surgery as being at high risk of postsurgical 
complications or poor outcomes. A multidisciplinary advanced care plan is then devised collaboratively with vists, surgical case managers and other relevant clinicians. This process is designed to ensure treatments are in keeping both with patient goals and values and with what is medically appropriate. Similar models have been welcomed by stakeholders ${ }^{10}$ and shown to be beneficial for cardiac and cancer care. ${ }^{11}$

The proposed model, Patient-Centred Advanced Care Planning (PC-ACP), allows for more extensive planning and may lead to safer and more effective care. The PC-ACP process provides a framework for logical engagement and communication with the patient and between clinical teams. If the model of care is shown to be beneficial, it may lead to better outcomes for high-risk patients presenting for surgery. Our project will implement this decision process in an Australian hospital, initially as a pilot study where PC-ACP will be trialled for patients undergoing elective surgery in the fields of cardiac surgery, general surgery, vascular surgery and orthopaedics. patients, their families, surgeons, anaesthetists, intensi-

The PC-ACP intervention

Patients at high risk of poor surgical outcomes require extra care to safeguard their journey through the surgical process. Delivering high-quality and maximally effective care to these patients requires a multispecialty approach. The PC-ACP intervention (figure 1) consists of a new decision-making process that leads to a multidisciplinary advanced care plan for high-risk surgery patients. The decision tree constitutes a framework for engagement and collaboration, not just with clinicians, but also with patients and families. It is intended to explore patients' goals and values and facilitate discussion on whether surgery aligns with those goals. Early work with colleagues from cardiothoracic surgery suggests this approach provides an improved cohesive response and more effective care.

The process will be triggered by surgeons who identify patients who are 'operable' but at high risk of adverse events due to frailty, multiple comorbidities and/or terminal illness. Unlike the current surgical pathway, this initial decision will be followed by multidisciplinary discussions with patients and their families, making explicit

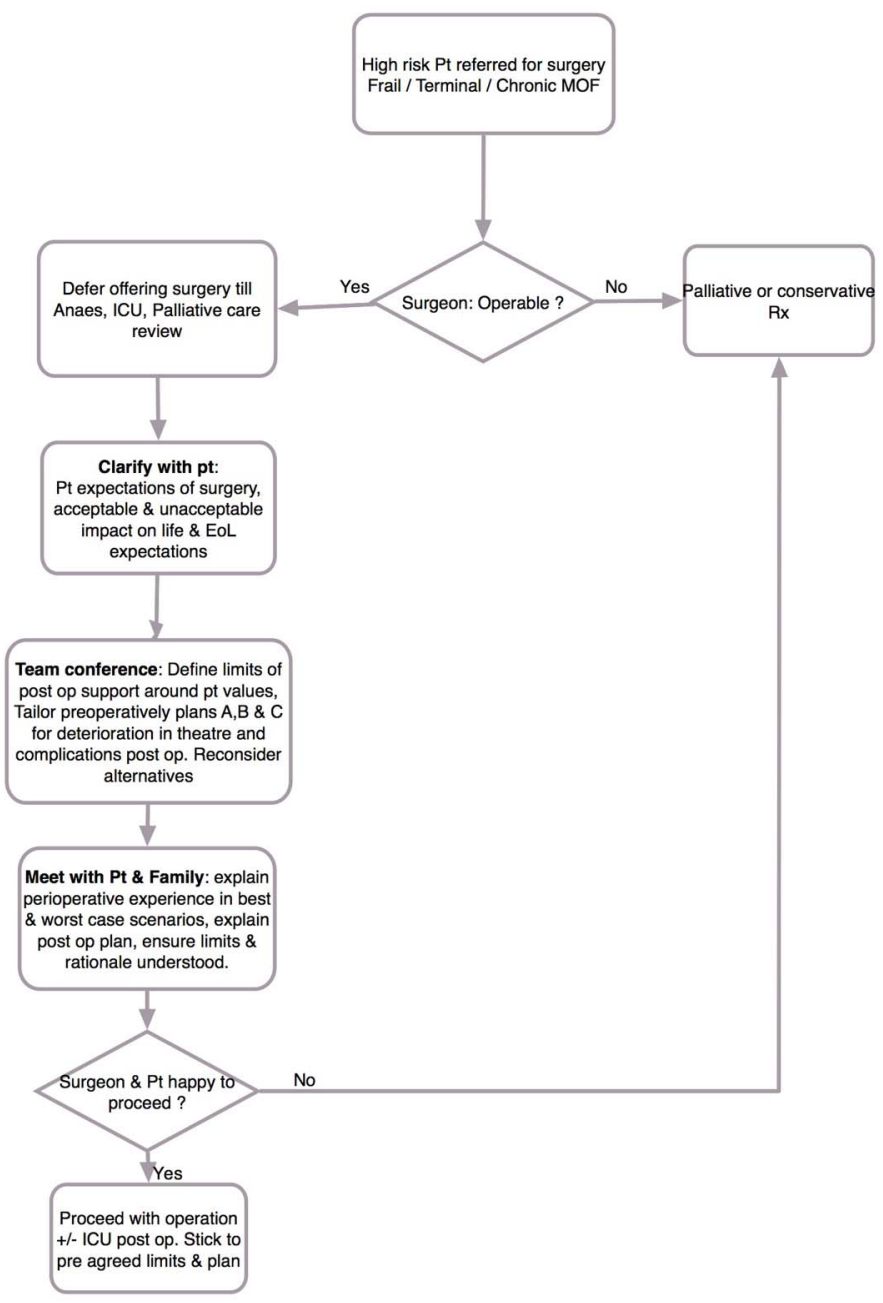

Figure 1 Patient-centred advanced care planning. EoL, end of life; ICU, intensive care unit; MOF, multiple organ failure. 
any surgical 'buy-ins', ${ }^{12}$ highlighting potential adverse outcomes of surgery and ensuring the patient is both clear about their choices and able to communicate their values prior to their decision to undergo surgery.

Changes in patients' preferences throughout their treatment can be accommodated within the PC-ACP framework as they communicate them; this process is not designed to elicit a rigid treatment plan. Instead, for patients and their families, it is designed to elicit a consideration and an understanding of the potential consequences of surgery and additional follow-up treatments. For clinicians, it is designed to elicit a shared understanding across disciplines of patients' values and goals so they can be acted on if necessary in circumstances when patients are unable to communicate their preferences.

\section{Project overview and scope}

This project aims to evaluate the feasibility of the new PC-ACP model of care and identify potential benefits to patients and clinicians. Results from this study will inform future guidelines for surgery on patients at high risk of adverse events or poor outcomes due to frailty, multiple comorbidities or terminal illness.

\section{METHODS AND ANALYSIS}

The study will involve several stages of surveys and interviews with both patients and staff (figure 2) and process mapping. We will use this triangulated qualitative and quantitative approach to determine the effectiveness of the new decision-making intervention. Two main types of data will be examined: patient experience and clinician experience. Patient experience data will also be used to map the patient journey (see Data analysis section).

\section{Study setting}

The study will take place at a public tertiary referral hospital in Queensland, Australia, at which PC-ACP will be implemented. Recruitment of participants and patient frailty assessments will take place within the relevant hospital departments. Surveys will be completed online or on paper at a convenient location. Interviews will be conducted at the hospital, participants' homes or over the phone, as participant circumstances permit.

\section{Participants}

Participants will consist of high-risk surgical patients, nominated family members and staff members involved in treating these patients (surgeons, anaesthetists, intensivists and surgical case managers). Inclusion criteria for patients, family members and staff members are listed in table 1. Patients meeting the inclusion criteria will be invited to participate in the study, which involves responding to two surveys and participating in three interviews. Each patient will have the option of nominating a family member to participate in interviews, either alongside the patient, or on their behalf. Recruitment of patients and family members will continue until $\sim 10$ patients (or nominated family members) have completed all surveys and interviews. The study will include patients who receive the PC-ACP model of care, regardless of their decision to proceed with, delay or decline surgery. Including patients who decline surgery as well as those who proceed with it will allow for a wider range of patient experiences within the PC-ACP model of care.

All clinical staff members at the participating hospital, who meet the inclusion criteria (table 1), will be invited to complete two surveys. One survey will be conducted at the initiation of the study, and the other following implementation of the PC-ACP. After each survey, up to 15 responders will be invited to participate in a follow-up interview. Clinical participants will be drawn from four groups of staff: surgeons, anaesthetists, intensivists and surgical case managers. Interviewees will be purposively selected to ensure at least one member of each group is interviewed and will be continued until data saturation is reached.

\section{Recruitment}

\section{Patients (and/or families)}

Surgeons working in the vascular surgery, cardiac surgery, general surgery and orthopaedics departments will be invited via letter to identify patients who meet the inclusion criteria (table 1) and refer them to investigators. The investigators will verbally inform these patients and their families about the research and provide written information about the study to those who are interested (see figure 2, top orange box). Patients and family members will be informed of the voluntary nature of their involvement, and that nonparticipation will not affect their medical care or their relationship with the hospital. They will be provided with a copy of the participant information sheet. Those who elect to participate will be assessed for frailty and invited to complete a survey either on paper or online. Patients completing the survey on paper will be given a paper consent form and survey and patients completing the survey online will be given a link for consent and survey completion. Both paper and electronic consent forms will include tick boxes via which participants can indicate their consent to be contacted for three semistructured interviews and a 6-month follow-up survey.

Patients who consent to be contacted will then be invited to participate in a series of three interviews. If the patient is unable to participate due to illness or incapacity, the family member responsible for their care decisions may elect to be interviewed on their behalf. Patients may also choose to have a family member participate alongside them in their interview. The family member approached will be the person named on the patient's advance care directive or hospital paperwork as next-of-kin or legal guardian (table 1).

\section{Staff members}

The investigators will verbally inform staff members who meet the inclusion criteria (table 1) about the research 


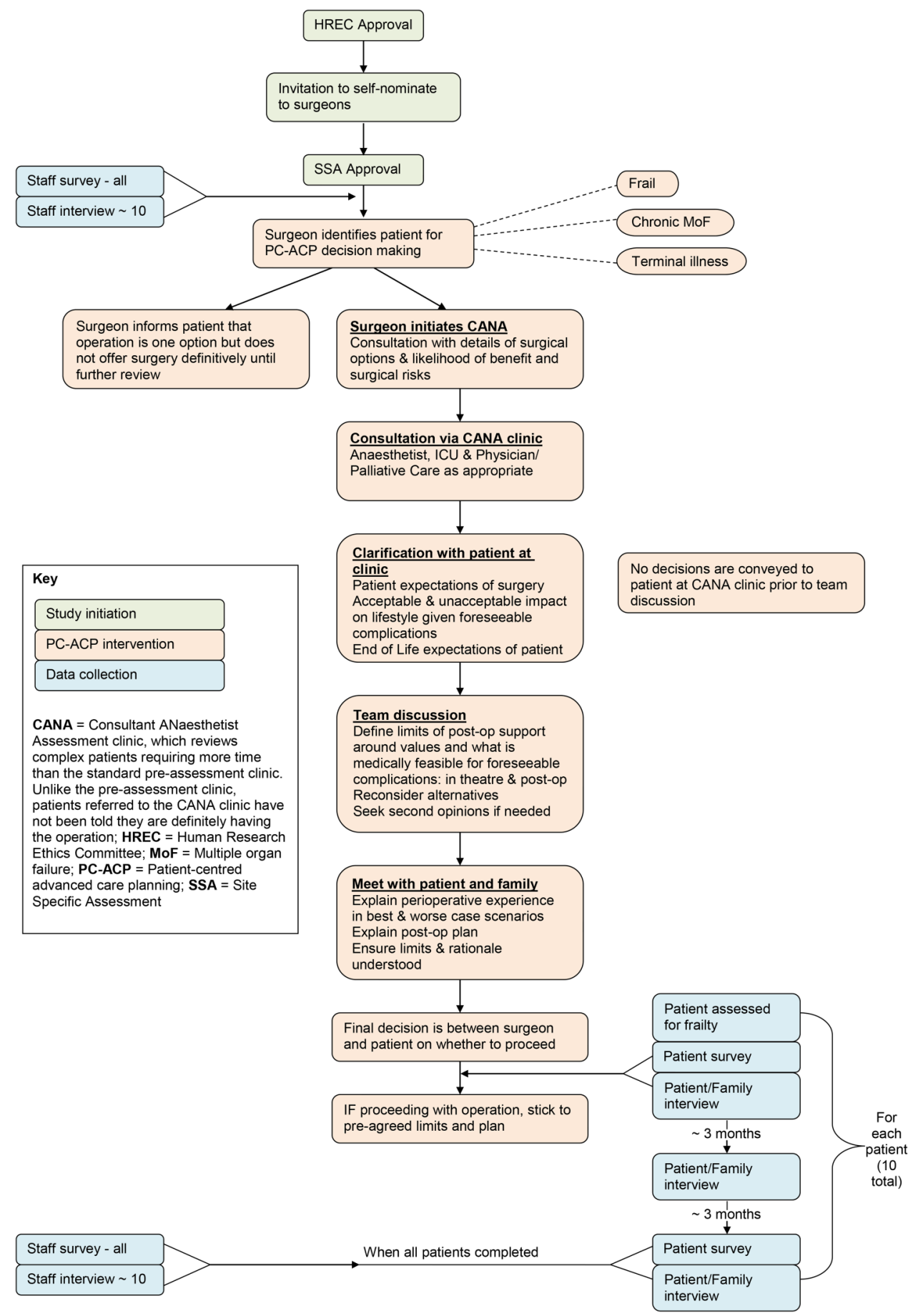

Figure 2 PC-ACP study outline. Green boxes=study initiation. Orange boxes=PC-ACP intervention. Blue boxes=data collection. CANA, Consultant ANaesthetic Assessment clinic, which reviews complex patients requiring more time than the standard preassessment clinic. Unlike the preassessment clinic, patients referred to the CANA clinic have not been told they are definitely having the operation; HREC, Human Research Ethics Committee; ICU, intensive care unit; MoF, multiple organ failure; PC-ACP, Patient-Centred Advanced Care Planning; SSA, site-specific assessment.

and provide written information about the study to those who are interested. Staff members will be informed of the voluntary nature of their involvement, and that non-participation will not affect job performance appraisal. They will be provided with a copy of the participant information sheet. Those who elect to participate will be invited to complete an online survey and given a link for consent and survey completion. The consent form will include tick boxes with which participants can indicate their consent to be contacted for a follow-up interview, a 6-month follow-up survey and a 6-month follow-up interview.

Up to 15 staff members who complete the survey and consent to be contacted for follow-up will then be 


\begin{tabular}{|c|c|}
\hline Patients & $\begin{array}{l}\text { Patient presenting for surgery with one } \\
\text { or more of the following: two or more } \\
\text { comorbidities, terminal illness, frailty } \\
\text { identified by treating clinician }\end{array}$ \\
\hline $\begin{array}{l}\text { Patient family } \\
\text { members }\end{array}$ & $\begin{array}{l}\text { Nominated by patient as next-of-kin or } \\
\text { surrogate for patient care } \\
\text { decision-making in advance care } \\
\text { directive or similar }\end{array}$ \\
\hline Staff members & $\begin{array}{l}\text { A minimum of } 50 \% \text { of time working as } \\
\text { surgical case manager (including } \\
\text { nursing staff), surgeon, anaesthetist, } \\
\text { intensivist, etc, for high-risk surgical } \\
\text { patients }\end{array}$ \\
\hline
\end{tabular}

invited to participate in a semistructured interview (figure 2, blue boxes at top left). Investigators will purposively select potential interviewees, using demographic survey data to ensure all groups of staff are represented in the interview sample.

At 6 months postimplementation, participating staff members who consent to be contacted will be invited to participate in a postimplementation survey and up to 15 survey responders will again be selected for follow-up interviews (figure 2, blue boxes at bottom left). Recruitment and selection will follow the same procedure as the initial survey and interview.

\section{Data collection}

Data collection will involve both quantitative and qualitative methods, with two major components: patient experience and clinician experience. Each component will involve several surveys and interviews over a 6 -month period.

\section{Patient frailty assessment}

Surgeons will refer patients they deem to be frail to this study. However, the participating hospital has no standard method of assessing frailty, and each surgeon may base their assessment on different criteria. Thus, investigators will independently assess the frailty of all participating patients using the Edmonton Frail Scale. ${ }^{13}$ This scale has been shown to be a valuable tool for assessing frailty in high-risk surgical patients ${ }^{14}{ }^{15}$ and the British Geriatrics Society recommends its use to assess frailty in all older patients presenting for elective surgery. ${ }^{16}$ The scale consists of 10 domains, each scored with 0,1 or 2 points. Two domains are practical: cognition, which involves drawing a clock with the hands pointed to a designated time, and functional performance, which measures how long the patient takes to stand up from a chair, walk $3 \mathrm{~m}$ and return to sit in the chair. The remaining domains consist of questions about general health status, functional dependence, social support, medication use, nutrition, mood and continence. Scores for each domain are added to give a total frailty score out of 17 , where $0-5=$ not frail, $6-7=$ vulnerable, $8-9=$ mild frailty, $10-11=$ moderate frailty and $12-17=$ severe frailty.

\section{Patient experience}

Patient experience will be measured using the Patient Clinician Information Engagement (PCIE) survey ${ }^{17}$ pretreatment and at 6 months follow-up (figure 2, blue boxes at bottom right). This validated survey examines patients' feelings of being informed about their treatment and information engagement between the patient and their clinician. This survey has demonstrated that feeling informed at baseline positively predicts decision satisfaction for patients at 12 months follow-up. ${ }^{17}$ The pretreatment PCIE survey consists of 13 fixed response components. The first five questions, measured by a fivepoint Likert scale from 1 (strongly disagree) to 5 (strongly agree) ask about patients' feelings of being informed. The following eight yes/no questions ask about patients' information seeking and engagement with their clinician in the first few months after their diagnosis of the condition requiring surgery. The posttreatment PCIE survey consists of two five-point Likert scale questions about patients' feelings of being informed, followed by eight yes/no questions about patients' information engagement in the past 6 months after their diagnosis. Both patient surveys will also collect information on basic demographics (age, gender, education and living arrangements) and the proposed surgery. Participants will have the option of completing the PCIE on paper or online using Qualtrics (Qualtrics software [program]. Provo, Utah, USA: Qualtrics, 2005. https://www.qualtrics.com/).

Patient experience will also be explored via three semistructured interviews. These interviews will be with patients and/or their nominated family member throughout their journey through the surgical process, at pretreatment and 3 and 6 months follow-ups (figure 2 , blue boxes at bottom right). The patient interview schedule is informed by the Cambridge University Hospitals National Health Service (NHS) Foundation Trust question set and guidance for patient feedback interviews. $^{18}$ Interviewees will be asked about the patient's journey through the surgical care pathway and their level of satisfaction with the processes of care. Patient semistructured interview questions are presented in online supplementary appendix A.

After consent has been obtained, a mutually convenient time will be arranged with each participant for a face-to-face interview in a private room. If a face-to-face interview is impractical, the participating patient or family member may be interviewed over the telephone. Interviews will be audio recorded for transcription. Before starting the interview, the interviewer will remind participants about the implications of their consent and that they can ask to stop the audio recording at any time. Depending on participant responses, it is anticipated that each patient interview will take up to $60 \mathrm{~min}$.

\section{Clinician experience}

Clinician experience will be measured using a revised version of the Safety Attitudes Questionnaire (SAQ) ${ }^{19}{ }^{20}$ 
at preimplementation and postimplementation of the new model of care (figure 2, blue boxes at top and bottom left). Over the last decade, the SAQ has emerged as an accepted standard for measuring clinician attitudes and engagement in the workplace. There are several versions of the survey, each optimised to a particular healthcare work environment. We will use the version specifically developed for the operating theatre (OR). The SAQ begins with a collaboration and communication section in which participants rate the quality of communication and collaboration with various hospital staff (eg, surgical staff, OR nurses), measured on a fivepoint Likert scale from 1 (very low) to 5 (very high). The remainder of the SAQ consists of 58 questions measuring attitudes in six domains (teamwork climate, safety climate, job satisfaction, stress recognition, perceptions of management and working conditions), using a five-point Likert scale from 1 (disagree strongly) to 5 (agree strongly).

In the revised version of the SAQ (OR version) used in this study, communication and collaboration ratings have been reduced from 15 to 9 . Ratings for irrelevant or inapplicable hospital staff have been removed or amended to fit with the Australian healthcare system, and ratings for patients and patients' families have been added to satisfy the study aims. In addition, the clinician survey only includes the three SAC domains relevant to this study: teamwork climate, safety climate and job satisfaction. Revising the survey in this manner maintains the integrity and validity of the tool while ensuring it collects data only on the aspects of team functioning relevant to the study. The revised survey is approximately half the length of the original, with 26 domain questions instead of the original 58. From previous experience with this instrument, we estimate that it will take clinicians not more than $10 \mathrm{~min}$ to complete the survey. The clinician survey will also collect information about clinicians' demographics (eg, age, gender) and professional experience (eg, position, years in specialty). Participants will complete the survey online using Qualtrics (Qualtrics software [program]. 2005).

Clinician experience with the PC-ACP will also be explored via semistructured interviews following each survey with up to 15 survey responders, or until data saturation is reached. Clinical participants will be asked about their experiences of applying the new decisionmaking process in their workplace and barriers or enablers to its implementation. The procedure for clinician interviews will match the procedure for face-to-face patient interviews. Depending on participant responses, it is anticipated that each staff interview will take up to $30 \mathrm{~min}$. Clinician semistructured interview questions are presented in online supplementary appendix B.

\section{Data analysis}

Survey data

All data will be de-identified and coded for analysis. Patients' frailty score from 0 to 17 on the Edmonton
Frail Scale ${ }^{13}$ will form part of patient pretreatment demographic data. All demographic data will be analysed via descriptive statistics and will be taken into account in the analysis of survey and interview data.

Pretreatment and 6-month follow-up patient surveys will be analysed and compared as per published procedures ${ }^{17}$ and via descriptive statistics to identify changes in patient satisfaction and feelings of being informed, and to determine whether pretreatment information seeking and engagement with clinicians predicts later satisfaction. Patient survey data will also inform process mapping. Patient frailty scores and patient demographics, such as details of proposed surgery or medical condition, will be included in survey analysis to discover whether these factors relate to any changes in patient goals and satisfaction from pretreatment to 6 months follow-up.

Clinician surveys will be analysed as per published procedures for the $\mathrm{SAQ}^{1920}$ and via descriptive statistics. Preimplementation and postimplementation surveys will be compared using statistical t-tests. This analysis will help ascertain clinician satisfaction with the PC-ACP model of care and identify any changes to workplace communication and culture resulting from the new model of care. Clinician demographics, such as professional role and experience, will be included in the analysis of clinician survey data to determine whether these factors relate to changes in workplace communication and culture as a result of PC-ACP implementation.

\section{Interview data}

Patient, family member and clinician interviews will be transcribed verbatim for analysis. Key themes from each sample will be identified by inductive interpretive analysis using the "constant comparative method'. ${ }^{21}$ This method will be somewhat modified to allow for the semistructured nature of the interview data. Using this method of coding, we will organise the interview data into data segments, which will then be formally linked. This process will allow themes to emerge and reveal potential relationships between data sets. This method will permit us to probe this real-world, complex system using multifaceted qualitative data from interviews. Coded patient and family member interview data will inform process mapping.

Clinician interviews will be coded with particular focus on barriers and enablers associated with implementing PC-ACP. These will be analysed according to the Theoretical Domains Framework. ${ }^{22}$ This framework for understanding behaviour change was developed to evaluate and inform implementation and has been successfully used in the context of healthcare. It includes 14 domains, each with several component constructs: knowledge; skills; social/professional role and identity; beliefs about capabilities; optimism; beliefs about consequences; reinforcement; intentions; goals; memory, attention and decision processes; environmental context and resources; social influences; emotion and behavioural regulation. ${ }^{22}$ Coding clinician interview data in 
line with this framework will ensure a complete account of barriers and enablers to implementation is obtained. Without this theoretical guidance, some less obvious but important barriers or enablers may be missed.

\section{Mapping the patient journey}

Process mapping ${ }^{23}$ will be used to map the journey of each patient through the surgical care pathway. This technique aims to identify the main components of the process, any critical or leverage points for process improvement and the extent to which the process varies between patients. Visualising the process helps identify process inefficiencies (eg, parallel or redundant processes) that are barriers to providing coordinated patient care. We will map the processes comprising the patient journey through the new PC-ACP model of care, based on de-identified data from the three stages of patient interviews and pretreatment and 6-month follow-up patient surveys. In this way, patient data will be used to develop a narrative outline of steps within the PC-ACP process and construct flow charts outlining the main stages of the patient journey.

\section{ETHICS AND DISSEMINATION}

This study is considered to be low risk for participants. Surveys and interview questions are not anticipated to be controversial or overly intrusive. Ethical approval has been obtained from the Townsville Hospital and Health Service Human Research Ethics Committee (HREC) associated with the participating hospital (HREC/16/ QTHS/100; figure 2, first green box), and hospital governance approval has also been obtained (SSA/16/ QTHS/193; figure 2, third green box). The data will be stored in a re-identifiable form until the completion of data collection, with a unique code for each participant. Findings will not be reported in a manner that enables individual participant responses to be identified. If groups (eg, surgeons) consist of fewer than five members, responses will be combined with those from other groups in reports.

Given the high risk of adverse events in the patient population involved in this study, one major ethical consideration is the possibility of distressing families of participants by contacting patients who have died during their treatment. To avoid this situation, investigators will contact the hospital to establish that the patient is alive, prior to contacting him or her at 3 and 6 months follow-up. In the event of patient's death, study investigators who are clinicians at the participating hospital will approach family members and invite them to participate in interviews. Since these investigators are experienced at communicating with families of deceased patients, this method of approaching family members will help safeguard their dignity. Interviewing family members about the patient's experience of care may be helpful to the grieving process. Nevertheless, this procedure will be carefully managed to ensure it will not be upsetting for families. Family members will only be invited for interviews, not the follow-up patient survey.

Results of this study will outline levels of patient satisfaction with PC-ACP, specify barriers and enablers to implementation and highlight critical points in the process for improvement. At the end of the project, draft and final evaluation reports will be disseminated to the participating hospital. These reports will present key findings from the study and recommendations for ongoing implementation of PC-ACP in high-risk surgical patients. Macquarie University investigators will present the findings and implications of the study to the hospital executive so that implementation of PC-ACP at the site can be optimised. Study findings and implications of interest to an academic audience will be submitted for publication in peer-reviewed MEDLINE-indexed journals and presented at academic conferences and workshops.

\section{CONCLUSION}

By introducing patient-centred decision-making to the surgical process, the new model of care has the potential to enhance the patient's experience and improve the journey through the surgical process for patients at high risk of poor outcomes. Under the new model, clinicians will share responsibility for health outcomes with patients, and patients will only be admitted for surgery if it meets their goals and values better than alternative treatments. Therefore, the new process may help prevent potentially avoidable hospital admissions. Seeking feedback from patients, family members and clinicians involved with the new model of care means the future implementation of this model will meet patients' needs while also mitigating barriers and profiting from enablers to implementation. Thus, this study will facilitate the best future implementation of PC-ACP.

\section{Author affiliations}

${ }^{1}$ Centre for Healthcare Resilience and Implementation Science, Australian Institute of Health Innovation, Macquarie University, Sydney, New South Wales, Australia

${ }^{2}$ Townsville Hospital and Health Service, Douglas, Queensland, Australia ${ }^{3}$ College of Medicine \& Dentistry, James Cook University, Townsville, Queensland, Australia

${ }^{4}$ Princess Alexandra Hospital, Yeronga, Queensland, Australia

Contributors SS conceived and developed the PC-ACP model of care. RC-W and SS devised the study design, protocol and plan and contributed to the development of the manuscript. AS contributed to the study design and protocol, led the ethics applications and led the development of the manuscript. BB contributed to the study design and the development of the manuscript. PL contributed to the study design and planning. JN provided clinical expertise and advice. SS and PL will provide on-site management of the study. RC-W, AS and BB will be involved in data collection and analysis. All authors have read and approved the final version of this manuscript.

Funding This work was supported by Townsville Hospital and Health Service Research Trust Fund grant number RG/2015/21.

Competing interests None declared.

Ethics approval Townsville Hospital and Health Service Human Research Ethics Committee.

Provenance and peer review Not commissioned; externally peer reviewed. 
Data sharing statement Data will be made publicly available to the extent that individual participants or participating hospitals cannot be identified, in accordance with requirements of the approving Human Research Ethics Committees.

Open Access This is an Open Access article distributed in accordance with the Creative Commons Attribution Non Commercial (CC BY-NC 4.0) license, which permits others to distribute, remix, adapt, build upon this work noncommercially, and license their derivative works on different terms, provided the original work is properly cited and the use is non-commercial. See: http:// creativecommons.org/licenses/by-nc/4.0/

\section{REFERENCES}

1. Bateni SB, Meyers FJ, Bold RJ, et al. Current perioperative outcomes for patients with disseminated cancer. J Surg Res 2015;197:118-25.

2. Kim W, Song KY, Lee HJ, et al. The impact of comorbidity on surgical outcomes in laparoscopy-assisted distal gastrectomy: a retrospective analysis of multicenter results. Ann Surg 2008;248:793-9.

3. Beggs T, Sepehri A, Szwajcer A, et al. Frailty and perioperative outcomes: a narrative review. Can J Anaesth 2015;62:143-57.

4. Lee DH, Buth KJ, Martin BJ, et al. Frail patients are at increased risk for mortality and prolonged institutional care after cardiac surgery. Circulation 2010;121:973-8.

5. Makary MA, Segev DL, Pronovost PJ, et al. Frailty as a predictor of surgical outcomes in older patients. J Am Coll Surg 2010;210:901-8.

6. Partridge JS, Harari D, Dhesi JK. Frailty in the older surgical patient: a review. Age Ageing 2012;41:142-7.

7. Redmann AJ, Brasel KJ, Alexander CG, et al. Use of advance directives for high-risk operations: a national survey of surgeons. Ann Surg 2012;255:418-23.

8. Williams SP, Howe CL. Advance directives in the perioperative setting: managing ethical and legal issues when patient rights and perceived obligations of the healthcare provider conflict. $J$ Healthc Risk Manag 2013;32:35-42.

9. Cardona-Morrell M, Kim J, Turner RM, et al. Non-beneficial treatments in hospital at the end of life: a systematic review on extent of the problem. Int J Qual Health Care 2016;28:456-69.
10. Schuster ALR, Aslakson RA, Bridges JFP. Creating an advance-care-planning decision aid for high-risk surgery: a qualitative study. BMC Palliat Care 2014;13:32.

11. Song MK, Kirchhoff KT, Douglas J, et al. A randomized, controlled trial to improve advance care planning among patients undergoing cardiac surgery. Med Care 2005;43:1049-53.

12. Schwarze ML, Bradley CT, Brasel KJ. Surgical "buy-in": the contractual relationship between surgeons and patients that influences decisions regarding life-supporting therapy. Crit Care Med 2010;38:843-8.

13. Rolfson DB, Majumdar SR, Tsuyuki RT, et al. Validity and reliability of the Edmonton Frail Scale. Age Ageing 2006;35:526-9.

14. Dasgupta M, Rolfson DB, Stolee $\mathrm{P}$, et al. Frailty is associated with postoperative complications in older adults with medical problems. Arch Gerontol Geriatr 2009;48:78-83.

15. Gordon A, Woodard J, Morris R, et al. Evaluating the Edmonton Frail Scale as a screening tool for postoperative complications in older patients undergoing elective hip and knee surgery. Eur Geriatr Med 2011;2(Supplement 1):S22.

16. British Geriatrics Society in association with the Royal College of General Practice and Age UK. Fit for frailty-consensus best practice guidance for the care of older people living in community and outpatient settings. London, UK: British Geriatrics Society, 2014

17. Martinez LS, Schwartz JS, Freres D, et al. Patient-clinician information engagement increases treatment decision satisfaction among cancer patients through feeling of being informed. Patient Educ Couns 2009;77:384-90.

18. The Health Foundation Co-Creating Health Programme. Agenda setting \& action planning: question set and guidance for patient feedback interviews. Cambridge University Hospitals NHS Foundation Trust, 2012.

19. Makary MA, Sexton JB, Freischlag JA, et al. Operating room teamwork among physicians and nurses: teamwork in the eye of the beholder. J Am Coll Surg 2006;202:746-52.

20. Sexton JB, Helmreich RL, Neilands TB, et al. The Safety Attitudes Questionnaire: psychometric properties, benchmarking data, and emerging research. BMC Health Serv Res 2006;6:44.

21. Glaser BG. The constant comparative method of qualitative analysis Soc Probl 1965;12:436-45.

22. Cane J, O'Connor D, Michie S. Validation of the theoretical domains framework for use in behaviour change and implementation research. Implement Sci 2012;7:37.

23. Damelio R. The basics of process mapping. Florida, USA: CRC Press, 2011. 